\title{
TURBULENCE IN INTERSTELLAR CLOUDS
}

\author{
E. Falgarone $e^{1,2}$ \\ ${ }^{1}$ Califormia Institute of Technology, 405-47, Pasadena, CA91125, USA \\ ${ }^{2}$ Radioastronomie, Ecole Normale Supérieure, \\ 24 rue Lhomond, 75235 Paris Cedex 05, France
}

\begin{abstract}
Above masses of the order of $100 M_{\odot}$, molecular clouds have masses and sizes which scale like those of self-gravitating polytropes bounded by an external pervading pressure. It is unlikely that this scaling is due to mere observational bias. But the physics underlying this behaviour is far from being understood. In particular, the possible contribution of turbulence to both the ambient pressure and the intemal pressure (whose dependence with the density would mimic a polytropic behavior) is a difficult and much debated issue. The clouds mass, size and internal velocity dispersion are such that they are observed to be in approximate virial balance between their self-gravity, the surface energy term due to the ambient pressure and their internal energy. The latter is dominated by the kinetic energy of disordered intemal motions. However, there has been little evidence so far that these motions are actually turbulent rather than simply disordered. The transition to turbulence in a flow occurs when the non linear advection term in the momentum equation, $v . \nabla v$, considerably exceeds the viscous dissipation term, $\nu \Delta \mathbf{v}$ (where $\nu$ is the kinematic viscosity). Non linearities therefore dominate the physics of a turbulent flow and the velocities are not randomly distributed. Most of the previous attempts to determine a well-defined correlation length in the velocity field (Kleiner and Dickman 1985, $a$ and $b$; Scalo 1984), which is predicted to be close to the scale at which the energy is injected, or to characterize the expected hierarchical structure (Pérault et al. 1986) have been plagued by the lack of dynamical range in the data set and the range of scales over which the correlation functions have been computed. The most plausible determination, that of Kleiner and Dickman (1987) who claim to have found a correlation length of $0.2 p c$ in the Taurus cloud, gives a result which is so close to the angular resolution of the observations that it is doubtful.
\end{abstract}

I choose here not to review the various attempts discussed in Dickman (1985) nor to cover the topics of the sources and sinks of turbulence in molecular clouds, these topics having been reviewed quite extensively by Scalo (1987) and Falgarone and Puget (1988). Instead, I will discuss the possible observational signatures of turbulence in the interiors of clouds. One of them is the element of surprise in the time history of the velocity field, in other words the existence of very localized regions of enhanced ephemeral vorticity which reflects the familiar and conspicuous intermittency in space and time of the turbulent activity. I will make ample use of results of measurements on laboratory flows and in the atmosphere and compare them with observations of interstellar clouds. Molecular clouds directly interacting with energetic events like the expansion of supemovae remnants and HII regions are not considered in what follows, even though those events count as a major source of kinetic energy and momentum in interstellar turbulence. 
The first part of this review provides partial answers to the question: are the disordered motions within interstellar clouds turbulent? Then, considering that there exist a few robust indications that turbulence is indeed present, the second part deals with possible important consequences of the turbulent nature of the gas motions to the physical and chemical evolution of the clouds.

\section{Are the internal motions in interstellar clouds turbulent?}

The maps of Figure 1 illustrate the intrinsic difficulty of defining a cloud edge without specifying the linear scale it is referred to. It displays the projected shapes at two different scales of the edges of a molecular cloud in the Taurus complex. The area selected in the large scale map of Ungerechts and Thaddeus (1987) for observations at higher angular resolution (Falgarone and Phillips 1989) has an average colomn density $N_{\mathrm{K}_{2}} \sim 910^{20} \mathrm{~cm}^{-2}$ (using the galactic average conversion factor between ${ }^{12} \mathrm{CO}$ integrated brightness and $\mathrm{H}_{2}$ column density, $N_{\mathrm{H}_{2}} / W_{C O}=$ $2.610^{20} \mathrm{~cm}^{-2} /\left(\mathrm{K} \mathrm{km} \mathrm{s}^{-1}\right)^{-1}$, Bloemen et al. 1986). In the undersampled map of Figure $1 \mathrm{~b}$, islands of ${ }^{12} \mathrm{CO}$ $(\mathrm{J}=2-1)$ emission appear with a highly contrasted brightness distribution. A crude comparison of the range of $\mathrm{H}_{2}$ column densities in these two maps can be made by assuming that the integrated brightness ratio between the ${ }^{12} \mathrm{CO}(\mathrm{J}=2-1)$ and $(\mathrm{J}=1-0)$ lines ranges between 0.5 and 0.8 in the low density edges $\left(n_{\mathrm{H}_{2}}<10^{3} \mathrm{~cm}^{-3}\right)$. The first contour of Figure $1 \mathrm{~b}$ thus corresponds to an $\mathrm{H}_{2}$ column density comparable to that of the third contour of Figure 1a but is much more convoluted. The striking property of these maps is that their degree of convolutedness looks self-similar and it is tempting to use the tool of fractal geometry to try to characterize more quantitatively this kind of structure.

\subsection{The fractal geometry of the cloud edges.}

In his approach to fractal surfaces and curves, Lovejoy (1982) defines a fractal dimension $D$ which relates the perimeter $P$ of a closed curve to the area $A$ enclosed within $P$ by the relation $P \propto A^{D / 2}$. $D=1$ (resp. 2) for classical curves (resp. surfaces) like a circle (resp. sphere) and tends toward $D=2$ (resp. 3) for an extremely convoluted curve (resp. surface) which would fill the entire plane (resp. space). Lovejoy measured a fractal dimension $D=1.35 \pm 0.05$ for rain areas and clouds at scales ranging between $1 \mathrm{~km}$ and $10^{4} \mathrm{~km}$. Table I summarizes the results obtained by the same technique on the fractal dimension of the contours of a variety of tracers of column density of interstellar clouds. The dimension derived from ${ }^{12} \mathrm{CO}$ line observations, in which the ${ }^{12} \mathrm{CO}(\mathrm{J}=1-0)$ data set is from Ungerechts and Thaddeus (1987) and the ${ }^{12} \mathrm{CO}(\mathrm{J}=2-1)$ and $(\mathrm{J}=3-2)$ from Falgarone, Phillips and Walker (1989) takes into account the effect of the various angular resolutions of the maps. $N_{d}$ is the number of decades covered by the values of the perimeter $P$.

Table I

\begin{tabular}{lllll}
\hline Tracer & Field & $\mathrm{D}$ & $\mathrm{N}_{d}$ & Reference \\
\hline IRAS $100 \mu \mathrm{m}$ & HLCs & $1.26 \pm 0.04$ & 2 & Bazell and Désert (1988) \\
IRAS $100 \mu \mathrm{m}$ & Taurus & 1.4 & 2 & Scalo (private communication) \\
${ }^{12}$ CO lines & Taurus & $1.34 \pm 0.02$ & 4 & Falgarone et al. (1989) \\
HI $21 \mathrm{~cm}$ & HVCs & $\sim 1.6$ & & Wakker (private communication) \\
\hline
\end{tabular}




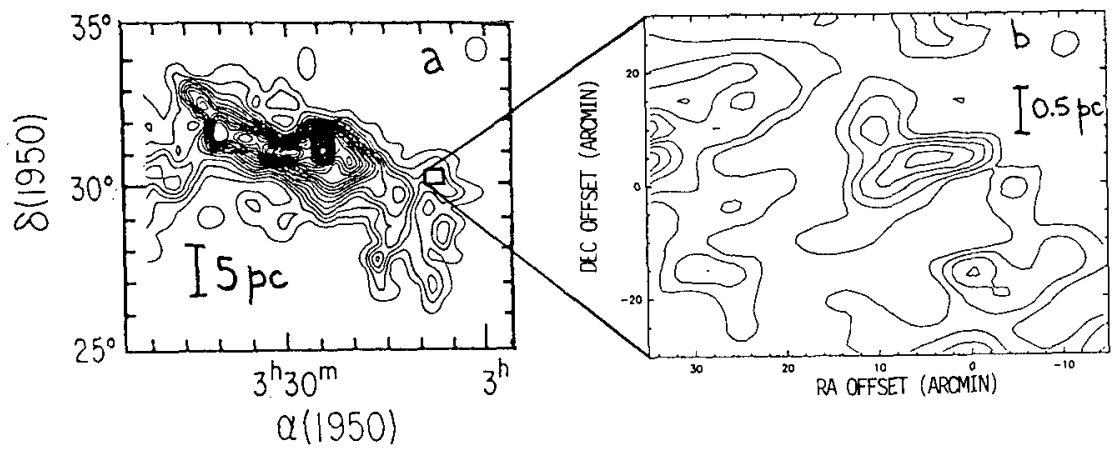

Figure 1. (a) Velocity-integrated intensity of ${ }^{12} \mathrm{CO}(\mathrm{J}=1-0)$ in the Taurus complex from Ungerechts and Thaddeus (1987). First contour $0.5 \mathrm{~K} \mathrm{~km} \mathrm{~s}^{-1}$, step $1.5 \mathrm{~K} \mathrm{~km} \mathrm{~s}^{-1}$. (b) Velocityintegrated intensity of ${ }^{12} \mathrm{CO}(\mathrm{J}=2-1)$ emission observed at the Caltech Submillimeter Observatory (HPBW=30", map sampled every 5'). First contour $2 \mathrm{~K} \mathrm{~km} \mathrm{~s}^{-1}$, step $2 \mathrm{~K} \mathrm{~km} \mathrm{~s}^{-1}$ (Falgarone et al. 1989).

The differences between the values of $D$ obtained with the different tracers of the gas column density may be real and significant but it is surprising that all the tracers provide values clearly larger than 1 . Indeed, the $100 \mu m$ brightness emission below a level of a few $10 \mathrm{MJy} \mathrm{sr}^{-1}$ is a tracer of the total column density of large dust grains and therefore of the total column density of $\mathrm{H}$ nuclei; ${ }^{12} \mathrm{CO}(\mathrm{J}=2-1)$ traces that of the $\mathrm{CO}$ molecules in the $J=2$ rotational level but may be considered as an indirect tracer of $\mathrm{H}_{2}$ column density in low brightness clouds; the Hl emission mapped by Wakker with the Westerbork interferometer is likely to be an exclusive tracer of the cold atomic component within the observed clouds. This suggests that there is no fundamental difference between the dominant physics which controls the shape of the edges of clouds, whether they are predominantly molecular or atomic.

The possible link with turbulence is the following. Sreenivasan and Meneveau (1986) and Meneveau (1989) have measured the fractal dimension of a variety of surfaces in turbulent flows. They do not measure the dimension of the projection of these surfaces onto a plane but that of the curve obtained by 2-dimensional slicing of a given interface. Their remarkable result is that, whether it is the interface between the vortex/no-vortex regions or the isoconcentration surfaces or the isodissipation surfaces, they find a similar dimension for the curve obtained by 2-dimensional slicing

$$
D^{\prime}=1.36 \pm 0.05
$$

There is no mathematical argument which clearly connects the dimension $D$ of the curve obtained by the projection on a plane of a fractal surface of dimension $D_{3 d}$ in the Euclidian space and that $D^{\prime}$ obtained by 2-dimensional slicing of the same surface by a plane, but limited experiments (Meneveau 1989) suggest that $D^{\prime} \leq D$. If isotropy is assumed, the fractal dimension of the surface itself is related to that of its projection by $D_{3 d}=D+1$.

Meneveau (1989) gives a simple physical argument for such a dimension. Let $\Phi(r)$ be the flux of any quantity $C$ which can be transported by turbulent diffusion across the surface element $S(r)$ of a fractal surface of dimension $D$ ( $r$ is the resolution element with which the surface and therefore the flux is actually measured). The turbulent 
diffusivity across the element of size $r$ is dominated by the motions at scale $r$ and is written $\nu_{t u r b} \sim r \Delta v(r) \sim r^{4 / 3}$, where $\Delta v(r) \sim r^{1 / 3}$, i.e. the average velocity difference between points separated by the distance $r$ is given by the Kolmogorov law. Then, $\Phi(r) \sim \nu_{\text {turb }} S(r) \nabla C$, where $\nabla C$, the gradient of the quantity $C$ over $r$, scales as $r^{-1}$. Hence, $\Phi(r) \sim S(r) r^{1 / 3}$. Since the value of $\Phi(r)$ should be independent of the value of $r$ used, $S(r) \sim r^{-1 / 3}$. The definition of the fractal dimension of a surface (Mandelbrot 1982) $S(r) \sim r^{2-D_{3 d}}$ then gives

$$
D_{3 d}=\frac{7}{3}=2.33 \text {. }
$$

The question of what is the quantity of importance which would be transported by turbulence across the cloud boundaries and therefore would regulate their shape and structure at all scales will not be addressed here. But it is to be roticed that this property shared by incompressible laboratory flows of high Reynold numbers and the atmosphere is not specific to turbulence. Similar fractal dimensions are found in the physics of critical phenomena (references in Meneveau 1989).

\subsection{Self-similarity of the velocity field.}

In 1941, Kolmogorov predicted the self-similar behavior of the velocity field in incompressible turbulent flows by postulating the existence of a local dissipationless cascade of kinetic energy from large scales to small scales which led him to state that the average velocity difference between two points separated by a distance $r$ should be a function only of $r$ and the energy dissipation rate per unit mass $\epsilon_{d}=\nu|\nabla \times \mathbf{v}|^{2}$. Dimensional arguments give the scaling with $r$ of the $n$-order moments of the increments of the velocity field

$$
M_{n}=<|v(\mathbf{x}+\mathbf{r}, t)-v(\mathbf{x}, t)|^{n}>=B_{n}\left(\epsilon_{d} r\right)^{n / 3},
$$

$B_{n}$ being universal constants. This prediction was later modified (Landau and Lifchitz 1959; Kolmogorov 1962) when laboratory experiments showed that the dissipation rate of specific kinetic energy was not uniform but concentrated in the limited regions of space and time where velocity gradients reach large values or diverge. A modified scaling was predicted to take into account the intermittency of $\epsilon_{d}$

$$
M_{n} \sim \bar{\epsilon}_{d} r^{\zeta_{n}}
$$

where $\bar{\epsilon}_{d,}$ is the average dissipation rate and $\zeta_{n} \neq n / 3$. Several theoretical approaches predict the form of $\zeta_{n}$ (see Section 1.3).

In real observations of interstellar clouds, the half-power width of a line profile is not simply related to the second order moment of the increment distribution of the velocity field (see Scalo, 1984). It is an average over the volume sampled by the telescope beam of the velocity difference between the most probable velocity $v_{o}$ (which is that of the peak temperature in optically thin lines) and all other velocities, the distance $r$ between two such points being subject to take all possible values between a minimum value which depends on the correlation length of the velocity field and the length of the line of sight through the cloud. It is easy to show that a beam of size $d \times d$ across a medium of size $l$ along the line of sight samples the increments of the velocity field over an average separation close to $d$ if the gas is uniformly distributed. The separation gets closer to $l$ when $l / d \geq 10$. This would explain why all the groups (Myers 1983; Leung, Kutner and Mead 1982; Dame et al. 1986; Solomon et 
al. 1987; Falgarone and Pérault 1987) which, after Larson (1981), have tried, using a variety of techniques, to relate the linewidth of molecular clouds $\delta v$ to their projected size $L$, do find a scaling law of the type $\delta v \sim L^{q}$. There is no consensus however upon the slope. For Larson (1981), $q=0.38$ while in the later investigations $q \sim 0.5$ for entities $L>0.2 p c$ up to $L \sim 100 p c$. Recent sensitive observations at high angular resolution have revealed complex structures as small as $0.02 p c$ within both opaque regions and low brightness envelopes (Pérault and Falgarone 1988). These entities, scen in the $(l, b, v)$ distribution of ${ }^{13} \mathrm{CO}$ or $\mathrm{C}^{18} \mathrm{O}$ emission as closed volumes of enhanced brightness (by a factor of $\sim 3$ above a smooth background emission) are found to be at best weakly bound by their self-gravity. Some of them have masses $\sim 10^{-2} M_{\odot}$, two orders of magnitude below the value for gravitational binding (Falgarone and Pérault 1988). When they are included in the cloud sample, the slope $q$ is quite close to $1 / 3$.

This is almost an embarrassing agreement with the law of Kolmogorov since interstellar clouds have highly compressible interiors likely to be threaded by magnetic fields. Equally embarrassing is the fact that self-gravitating and non self-gravitating entities are now included in the sample. But it is to be noticed again that self-similar laws are also characteristics shared by many non-linear dissipative systems.

\subsection{Intermittency of the velocity field}

Decades ago, Van der Hulst (1958) and Münch (1957) had already noticed from a statistical study of the velocity of the Call and NaI absorption lines in the interstellar medium that the distribution of these velocities was better approximated by an exponential than by a gaussian. An observational finding, which may be related to that is the detection by Shaver et al. (1982) and Anantharamaiah et al. (1984) of a long tail of HI absorption components spreading in the forbidden velocity range in the direction of galactic HII regions. This point is controversial and Kulkarni and Fich (1985) who detected this gas in emission argue that it is the in-the-plane counterpart of the galactic HI halo (Lockman 1984). A still more controversial point is the existence of non gaussian wings in the HI profiles of face-on galaxies (Lewis 1987; Dickey et al. 1989). Usually considered as tracers of HI warps due to tidal interaction with companions, it is conspicuous that in a few cases they are observed in isolated galaxies. In molecular clouds not associated with star forming activity, non gaussian wing excesses have been reported in several cases (Blitz and Stark 1986; Knapp and Bowers 1988; Magnani, Blitz and Wendel 1988). The latter show that the fast gas they detect has a column density which exceeds by 3 orders of magnitude that expected from the evaporation of the clouds in the hot interstellar medium.

In an attempt to study these excesses more systematically, Falgarone and Phillips (1989) have analyzed the line profiles of several rotational transitions of $\mathrm{CO}$ and variants over regions of sizes ranging between $0.02 p c$ and 450 pc. Profiles come from the literature (Scoville and Young 1983; Pérault et al. 1985; Blitz and Stark 1986; Magnani et al. 1988; Falgarone and Pérault 1988), and unpublished data from the IRAM-30m telescope, the Caltech Submillimetre Observatory and other telescopes (Ungerechts; Fukui, private communications). They find that most of them have wing emission above the level expected from a gaussian line core (e.g. in Figure 2 and in Falgarone and Phillips 1989) and that the ratio of the velocity width of the wing, treated as a second gaussian, to that of the core is remarkably constant throughout the sample,

$$
\frac{\sigma_{\text {wing }}}{\sigma_{\text {core }}} \sim 3
$$


This scaling makes it unlikely that the wing emission at all scales be due to a hot diluted component or protostellar outflows. Moreover, in two cases, Falgarone, Phillips and Walker (1989) show that the wing emission is beam diluted emission of small thermalized fragments. A tentative conclusion is that the wing emission is an intrinsic feature of the velocity field of molecular clouds at all scales, previously overlooked due to the good signal to noise ratio required to detect it. Assuming that the wing emission is a good tracer of the probability distribution of the velocity difference $v-v_{o}$ over the volume sampled by the beam, a possible attractive interpretation of the wing emission is that it is the signature of the intermittency of the velocity field within molecular clouds. It would reveal a higher than normal (gaussian) number of rare events, the rare events being velocity fluctuations of large amplitude about $v_{o}$.

A comparison with experimental results on duct flows at high Reynold numbers (Anselmet et al. 1984) and direct measurements in the atmosphere (Van Atta and Park 1971; Dutton and Deaven 1969) is very suggestive (Figure 2b). All the data show that the probability distribution function of the velocity increments present a significant excess at large values which is interpreted as due to the intermittency of the velocity field. They also find that the phenomenon of intermittency is as more pronounced as the scales are small but that neither the lognormal model
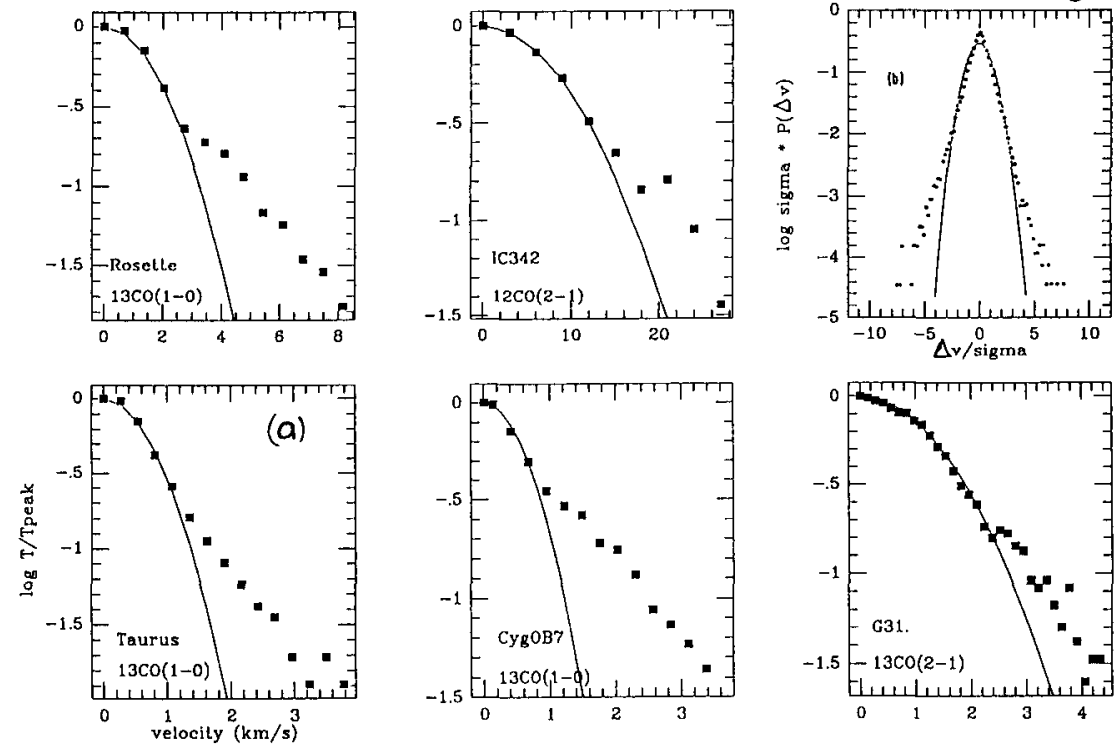

Figure 2. (a) Lin-log plot of a subset of normalized profiles (references in the text). The gaussian of same half power width is drawn. (b) Probability density of the velocity difference between two points separated by $r=4.4 \mathrm{~cm}$ from measurements in the atmospheric boundary layer (van Atta and Park, 1971).

(Kolmogorov, 1962) nor the $\beta$-model (Frisch et al. 1978) are able to reproduce the experimental data. In his multifractal approach to turbulence, Meneveau (1989) has recently been able to reproduced the values of Anselmet et al. (1984) up to the 18-order moment of the velocity probability distribution. Observations of interstellar clouds are orders of magnitude less sensitive than in situ measurements in duct flows and such a beautiful comparison with a statistical model is far beyond what can be achieved at the moment. But further sensitive observations and 
analysis of the lineprofiles should be able to decide upon the reality and possible ubiquity of this phenomenon. Spatial mapping of the wing emission should bring information on its nature at the different scales. If intermittency is due to non linear amplification of vorticity (Lundgren 1982), blue and red emission should be spatially correlated, at all scales. In any event, the observation of intermittency in the cloud velocity fields would provide a solid element to the proof of turbulence.

\subsection{Compressibility}

In 1951, Chandrasekhar and Münch derived the amplitude of the space density fluctuations in the interstellar medium from a statistical analysis of the fluctuations of the absorption of the galactic light along the plane. Using a few assumptions on the space distribution of the absorbing material, they derived from the survey data of Pannekoek and Koelbloed (1949), the root mean square amplitude of the density fluctuations $\left\langle\delta \rho^{2}\right\rangle^{1 / 2} \sim 3$ to $4\langle\rho\rangle$ $(\langle\rho\rangle$ is the average density) and proposed that the interstellar medium is a continuous distribution of density fluctuations of small amplitude rather than an ensemble of discrete clouds. Within molecular clouds, regions of enhanced density far from star forming cores cannot be described entirely as islands in a sea of low brightness emission but tend to be connected in space and velocity (Falgarone and Pérault 1988; Bally et al. 1987; Swade 1989). This result seems to be valid down to scales as small as $0.02 p c$ both within clouds areas of low average column density $\leq 10^{21} \mathrm{~cm}^{-2}$ and within the so-called dense cores (see Puget and Falgarone 1989).

It is interesting to note that Passot et al.(1988) find, on the basis of two dimensional simulations, that comparable density contrasts are compatible with a turbulence of low ms Mach number. The highly filamentary structures which develop in their density field are in addition very reminiscent of those observed in molecular clouds.

A provisional conclusion would then be that, on the basis of observations only, the velocity field within interstellar clouds is very likely to be turbulent with a moderate level of compressibility, somewhat smaller than would be expected from the crude but popular estimate $\left\langle\delta \rho^{2}>^{1 / 2} \sim M^{2}<\rho>\right.$ where $M \sim 3$ is a kind of Mach number of the turbulence equal to the ratio of observed velocities to the sound speed in clouds. The gas flow within clouds would present similarities to those observed in ducts or in the atmosphere, in particular the spatial and temporal intermittency of the velocity and velocity gradient (therefore of the dissipation rate of kinetic energy) and the fractal geometry of the cloud structure. We discuss below a few consequences of the presence of turbulence within clouds.

\section{The role of turbulence in the physics and chemistry of molecular clouds}

\subsection{The disputed issue of turbulent pressure}

It is repeatedly found in the literature (e.g. van Dishoeck and Black 1986) that intemal turbulent motions contribute locally to the gas pressure in clouds by an amount $\propto \rho \sigma_{v}^{2}$ where $\sigma_{v}$ is a velocity dispersion of the non thermal motions usually provided by the linewidths and is therefore related to the entire volume sampled by the beam. As long as only global balances are discussed this may be correct. The additional kinetic energy in turbulent motions can be viewed as a pressure term at the scale for example of gravitationally bound entities in which self-gravity plays the role of the containing walls for the mass of gas. In unbound clouds or locally, the concept of turbulent pressure is not even intuitive. Turbulent pressure is likely to be scale dependent and a lump of 
matter of size $L=2 \pi / K$ will be affected differently by motions at scales $k>K$ and $k \sim K$. The latter, which in most cases contain more energy than the former, permanently redistribute the mass contained within lumps of gas at scale $K$. The existence and the role of a turbulent pressure depends not only on the total power in turbulence but also on the slope of its power spectrum.

Turbulent pressure has also been invoked to play a role in the confinement of molecular clouds (Maloney 1988). If there is clearly some contribution of a surface term in the virial equilibrium of molecular clouds (Chièze 1987), the uncertainties on the parameters derived from observations and the weak sensitivity to the ambient pressure, $P_{0}$, which is predicted for the internal velocity dispersion of a self-gravitating polytrope $\sigma_{v} \propto P_{0}^{1 / 4} R^{1 / 2}$, make any

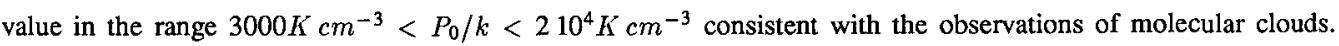
Such a broad range does not allow any estimate of the importance of a possible turbulent contribution to a pressure which would confine or help to confine interstellar clouds at all scales.

What observational data unambiguously show is that the large scale motions and the magnetic field are the two agents which prevent molecular clouds (which contain hundreds of Jeans masses) from collapsing in free fall (Myers and Goodman 1988a). However it is not straightforward to account theoretically for the global stability of clouds in which dense cores and stars form. The gradual loss of magnetic support in the densest and less ionized parts may explain the slow formation of dense cores (Shu et al. 1987; Myers and Goodman 1988b), but the support of the large scale and more unstable envelope has to be accounted for simultaneously. Turbulence may play a major role there.

\subsection{Gravitational instability in a turbulent medium}

The problem may be formulated as follows. In a medium of density $\rho_{0}$ and sound velocity $c_{s}$, density perturbations of wavenumber smaller than $K_{J}=\sqrt{4 \pi \rho_{0}} / c_{s}$ grow exponentially if the only support for the gas against gravity is thermal pressure. If, instead, the equilibrium state is a solution of the equation of motion with an adequate forcing which generates stationary turbulence, one may ask how the velocity field is going to react to the density perturbation at scale $K$ and if a gradient of turbulent pressure is going to develop to resist the gravitational forces over the unstable scales. Early attempts to solve this problem are due to Chandrasekhar (1951) and Sasao (1973) but neither of them demonstrates the existence of a turbulent pressure. Bonazzola et al.(1987) have shown in 2-dimensional numerical simulations that Jeans unstable scales can be stabilized by supersonic turbulence and that it is due to the non linear term in the equation of motion.

An analytical approach to this question is possible using renormalization group techniques (Bonazzola et al., 1989). They write an equation of motion for the velocity field built on Fourier components of spatial frequencies smaller than $K$. For linear density perturbations, this equation keeps the same form as the Navier-Stokes equation with pressure and viscosity terms renormalized to describe the dynamical effects of the small scales $(k>K)$. The form of the turbulent viscosity they find is a rather classical result (see for example Moffat, 1981). The original result of Bonazzola et al. (1989) is that a term proportional to the density gradient appears in the equation of motion (the turbulent pressure gradient) and is given by an integral of the power in turbulent motions over spatial frequencies larger than $K$. The contribution of the turbulent velocity field to the pressure is given by:

$$
V_{K}^{2}=\int_{K}^{+\infty} d k\left[\frac{2}{3} I^{\perp}(k)+A I^{\|}(k)\right]
$$


where $0 \leq A \leq \frac{1}{3}$ and $I^{\perp}(k)$ and $I^{\|}(k)$ are the power spectra of the correlations of the incompressible and compressible parts of the velocity field respectively. It should be noticed that the incompressible part of the velocity field contributes to the turbulent pressure in the same way as thermal motions of the particles to the kinetic pressure and that the same energy density in the form of a compressible field only would contribute less to the turbulent pressure.

If a linear gravitational stability analysis is carried out on a fluid controlled by this equation of motion, the following dispersion relation is derived:

$$
\omega^{2}-\left(c_{s}^{2}+V_{K}^{2}\right) K^{2}-4 \pi G \rho_{0}=0
$$

(the viscous terms have been omitted for clarity). The stabilisation effect of the turbulence is immediately visible since the pressure gradient term is increased. Furthermore, as shown by Bonazzola et al. (1987), if the turbulent spectrum is steep enough, the usual Jeans criterion might even be reversed, the largest scales being the most stable. The turbulent pressure is only one aspect of the possible effects of supersonic turbulence on gravitational instability. A full analysis must include the generation of non linear density fluctuations by the compressible velocity field and that of their lifetime. This analysis has not been done yet.

Recently, Léorat et al.(1989) have considered the same problem and their 2D numerical simulations show a stabilisation by supersonic turbulence (see also Passot, 1987) but their interpretation of this stabilisation is different from the previous one. They claim that the compressible part of the velocity field is the only one at work in the process. A major improvement in the understanding of the problems mentioned above will occur with $3 \mathrm{D}$ simulations including self-gravity over a large range of spatial frequencies.

\subsection{Consequences of turbulent mixing on chemical abundances}

Chièze and Pineau des Forets (1989) have attempted to introduce turbulent mixing in a chemical code following an idea of Boland and de Jong (1982). They picture a cloud as a massive envelope of low column density and space density which is exchanging mass with much denser a core completely shielded from the ambient UV field. The parameter of importance is the mixing time of the matter in the core $t_{\text {mix }}=M_{\text {core }}(d M / d t)^{-1}$ where $d M / d t$ is the mass exchange rate between the core and the envelope. Their remarkable result is that provided that $t_{\text {mix }}<10^{6} y r$ the abundances of both the neutral and ionized carbon in the dense core are enhanced by more than an order of magnitude above the value they would reach in classical models without mixing.

\section{Conclusions and prospective}

The self-similarity of the spatial and velocity structure of interstellar clouds, the plausibility of a Kolmogorovtype cascade of kinetic energy, the spatial intermittency of the velocity field and the existence of a turbulent pressure are all elements which, taken separately, might be the result of observational biases or attributed to any non linear process in the physics of the clouds. Taken all together, they point to the conclusion that turbulence does exist within clouds, be it purely hydrodynamic or rather hydromagnetic. The impact of such a conclusion to our understanding of the physics of the interstellar medium is yet to be determined. For example, all the processes govemed by the penetration of photons in the cloud interiors would be modified by a fractal topology. This would affect considerably the heating processes, the chemistry, the radiative cooling rates and therefore the equation of 
state. Turbulent pressure has been shown to play a major role in the stabilisation of clouds against gravity and therefore in the star formation process.

One should not feel discouraged by the apparent intractability of turbulence but rather encouraged by the following. The recent developments of powerful instruments provide the community working on the interstellar medium with an unprecedented amount of high quality data. Also, new theoretical approaches to chaos and unpredictable behaviour of non linear systems, in conjunction with the results of powerful simulations and remarkable laboratory experiments should provide tools or guidelines to handle the complexity of the data.

\section{References}

Anantharamaiah, K. R., Radhakrishnan, V., Shaver, P. 1984, Astr. Ap., 138, 131.

Anselmet, F., Gagne, Y., Hopfinger, E. J. 1984, J. Fluid Mech., 140, 63.

Bally, J., Langer, W.D., Stark, A.A., Wilson, R.W. 1987, Ap. J. Letters, 312, L43.

Bazell, D. and Désert, F. X. 1988, Ap. J., 333, 353.

Blitz L., Stark A.A. 1986, Ap. J.(Letters), 300, L89.

Bloemen, J.B.G.M. et al 1986, Astron. Astrophys., 139, 37.

Boland, W. and de Jong, T. 1982, Ap. J., 261, 110.

Bonazzola et al. 1987, Astron. Astrophys., 172, 293.

Bonazzola et al. 1989, submitted to Physics of Fluids.

Chandrasekhar, S. 1951, Proc. Roy. Soc. A., 210, 18 and 26.

Chandrasekhar, S. and Münch, G. 1951, Ap. J., 115, 103.

Chièze, J. P. 1987, Astr. Ap., 171, 225.

Chièze, J. P. and Pineau des Forets, G. 1989, Astr. Ap., in press.

Dame, T.M., Elmegreen, B.G., Cohen, R., Thaddeus, P. 1986, Ap. J, 305, 892.

Dickey, J. M., Hansen, M. M., and Helou, G., preprint.

Dickman, R. 1985, "Protostars and Planets II", eds. D. C. Black and M. S. Matthews, Univ. of Arizona Press.

Dutton, J. A. and Deaven, D. G. 1969, Radio Science, 4, 1341.

Falgarone, E., Pérault, M.: 1988, Astron. Astrophys., 205, L1.

Falgarone, E., Puget, J.L.: 1986, Astron. Astrophys., 162, 235.

Falgarone, E., Puget, J.L.: 1988, Galactic and Extragalactic Star Formation, eds. R.E. Pudritz and M. Fich. Falgarone, E., Pérault, M.: 1987, Physical Processes in Interstellar Clouds, eds. G.E. Morfill and M. Scholer. Falgarone, E., Phillips, T. G. 1989: Submillimetre and Submillimetre Astronomy, ed. A. Webster, in press

Falgarone, E., Phillips, T. G., Walker C. 1989, in preparation.

Frisch, U., Sulem, P. L., Nelkin, M. 1978, J. Fluid Mech., 87, 719.

Kleiner, S. C. and Dickman, R. L. 1985, Ap. J., 295, 466 and 479.

Kleiner, S. C. and Dickman, R. L. 1987, Ap. J,, 312, 837.

Knapp, G. R. and Bowers, P. F. 1988, Ap. J., 332, 299.

Kolmogorov A.N. 1941: Dokl. Akad. Nauk. 26, 115.

Kolmogorov, A. N. 1962, J. Fluid Mech., 13, 82.

Kulkami, S. R. and Fich, M. 1985, Ap. J., 289, 792.

Landau, L. D. and Lifchitz, E. M. 1959, Fluid Mechanics, Addison-Wesley.

Larson, R.B.: 1981, Monthly Notices Roy. Astron. Soc., 194, 809.

Léorat, J., Passot, T., Pouquet, A.: 1989, Astron. Astrophys., submitted.

Leung, C. M., Kutner, M. L., and Mead, K. N. 1982, Ap. J., 262, 583.

Lewis, B. M. 1987, Ap. J. Suppl., 63, 515.

Lockman, F. J. 1984, Ap. J., 283, 90.

Lovejoy, S. 1982, Science, 216, 185. 
Lundgren, T. S. 1982, Phys. Fluids, 25, 2193.

Magnani L., Blitz L., Wendel A. 1988, Ap. J.(Letters), 331, L127.

Maloney, P. 1988, Ap. J., 334, 761.

Mandelbrot, B. B. 1982, "The fractal geometry of nature", Freeman.

Meneveau 1989, PhD. dissertation, Yale University.

Meneguzzi, M., Frisch, U., Pouquet, A. 1981, Phys. Rev. Letters, 47, 1060.

Moffat, H.K.: 1981, J. Fluid Mech., 106, 27.

Münch, G. 1957, Ap. J., 125, 42.

Myers, P.C. 1983: $A p . J ., 270,105$.

Myers, P.C., Goodman, A.A. 1988a, Ap. J. Letters, 326, L27.

Myers, P.C., Goodman, A.A. 1988b, Ap. J., 329, 392.

Pannekoek, A. and Koelbloed, D. 1949, Pub. Astr. Inst. Amsterdam, 9.

Passot, T.: 1987, Thèse d'Etat Université Paris VII.

Passot, T., Pouquet, A. and Woodward, P. 1988, Astron. Astrophys., 197, 228.

Pérault M., Falgarone E., Puget J.L. 1985, Astron. Astrophys., 152, 371.

Pérault, M., Falgarone, E., Puget, J.L. 1986, Astr. Astrophys., 157, 139.

Pérault, M. and Falgarone, E.: 1988, Molecular Clouds in the Milky Way and External Galaxies, eds. $J$. Young and R. Snell.

Puget J.-L. and Falgarone, E. 1989: Submillimetre and Submillimetre Astronomy, ed. A. Webster, in press

Sasao, T. 1973, Publ. Astron. Soc. Japan, 25, 1.

Scalo, J. M. 1984, Ap. J., 277, 556.

Scalo, J.M., Pumphrey, W.A.: 1982, Ap. J.(Letters), 258, L29.

Scalo, J.: 1987, Interstellar Processes, eds. D.J. Hollenbach and H.A. Thronson.

Scoville, N. and Young, J. 1983, Ap. J., 265, 148.

Solomon, P.M., Rivolo, A.R., Barrett, J., Yahil, A. 1987: Ap. J., 319, 730.

Sreenivasan, K. R. and Meneveau, C. 1986, J. Fluid Mech., 173, 357.

Shaver, P. A., Radhakrishnan, V., Anantharamaiah, K.R., Retallack, D.S., Wamsteker, W., Danks, A.C. : Astr. Astrophys., 106, 105.

Shu, F., Adams, F.C., Lizano, S.: 1987, Annual Review of Astron. and Astrophys., 25, 23.

Swade, D. A. 1989, Ap. J. Suppl., in press.

Ungerechts, H. and Thaddeus, P. 1987, Ap. J. Suppl., 63, 645.

van Atta, C. W. and Park, J. 1971, "Statistical Models and Turbulence", eds. M. Rosenblatt and C. van Atta: Springer.

van der Hulst, H. C. 1958, Rev. Mod. Phys., 10, 913.

van Dishoeck, E. F. and Black, J. H. 1986, Ap. J. Suppl., 62, 109. 


\section{Discussion:}

SOLOMON: The exponent in the size-linewidth relation $\sigma_{V} \propto R^{\beta}$ is not 0.33 in either local dark clouds (Myers et al.) or in a sample of 270 galactic plane molecular clouds (Solomon et al., 1987). For both of these large samples covering a range in size from about $1 \mathrm{pc}$ to $100 \mathrm{pc}$, the exponent is 0.5 . Thus $\beta$ does not agree with the value expected for the Kolmogorov spectrum in gravitationally bound clouds.

FALGARONE: I am aware of this point since we also found a slope 0.5 in the $\sigma_{V}$ versus size relation for a sample of about 90 clouds or condensations observed in ${ }^{13} \mathrm{CO}$ (1-0) (Falgarone and Perault,1987). Only when we included unbound maxima of column density isolated from $(1, \mathrm{~b}, \mathrm{~V})$ maps of ${ }^{13} \mathrm{CO}$ and $\mathrm{C}^{18} \mathrm{O}$ emission, covering the range of sizes from $0.02 \mathrm{pc}$ to $0.2 \mathrm{pc}$, did we find that a slope closer to $1 / 3$ was clearly a better fit to the ensemble of data. This change of slope may be significant and there are many reasons to expect a change in the physics of the hierarchy around the parsec scale. But I just wanted to mention that a $1 / 3$ power law is not ruled out.

DICKEY: A Kolmogorov spectrum is ordinarily generated by a cascade of turbulent energy from large scales to smaller scales. Do you think such a thing is happening in the interestellar gas, or does some other process determine the slope of the spectrum?

FALGARONE: No, I don't think there is a cascade of energy. There are too many sources of turbulence on all different scales to tell how the energy flows. 